\title{
A systems biology approach to detect key pathways and interaction networks in gastric cancer on the basis of microarray analysis
}

\author{
LEILEI GUO, CHUNHUA SONG, PENG WANG, LIPING DAI, JIANYING ZHANG and KAIJUAN WANG \\ Department of Epidemiology and Biostatistics, College of Public Health, \\ Zhengzhou University, Zhengzhou, Henan 450001, P.R. China
}

Received November 6, 2014; Accepted July 31, 2015

DOI: $10.3892 / \mathrm{mmr} .2015 .4242$

\begin{abstract}
The aim of the present study was to explore key molecular pathways contributing to gastric cancer (GC) and to construct an interaction network between significant pathways and potential biomarkers. Publicly available gene expression profiles of GSE29272 for GC, and data for the corresponding normal tissue, were downloaded from Gene Expression Omnibus. Pre-processing and differential analysis were performed with $\mathrm{R}$ statistical software packages, and a number of differentially expressed genes (DEGs) were obtained. A functional enrichment analysis was performed for all the DEGs with a BiNGO plug-in in Cytoscape. Their correlation was analyzed in order to construct a network. The modularity analysis and pathway identification operations were used to identify graph clusters and associated pathways. The underlying molecular mechanisms involving these DEGs were also assessed by data mining. A total of 249 DEGs, which were markedly upregulated and downregulated, were identified. The extracellular region contained the most significantly over-represented functional terms, with respect to upregulated and downregulated genes, and the closest topological matches were identified for taste transduction and regulation of autophagy. In addition, extracellular matrix-receptor interactions were identified as the most relevant pathway associated with the progression of GC. The genes for fibronectin 1, secreted phosphoprotein 1 , collagen type 4 variant $\alpha-1 / 2$ and thrombospondin 1, which are involved in the pathways, may be considered as potential therapeutic targets for GC. A series of associations between candidate genes and key pathways were
\end{abstract}

Correspondence to: Professor Kaijuan Wang, Department of Epidemiology and Biostatistics, College of Public Health, Zhengzhou University, 100 Science Avenue, Zhengzhou, Henan 450001, P.R. China

E-mail: kjwang@163.com

Key words: graph clustering, pathway crosstalk, modularity analysis, interaction network, gastric cancer also identified for GC, and their correlation may provide novel insights into the pathogenesis of GC.

\section{Introduction}

Gastric cancer (GC) is one of the leading causes of cancer-associated mortality worldwide, and was the third leading cause of cancer-associated mortality in China in $2012(1,2)$. However, the etiology of GC is complicated and remains to be fully elucidated. Numerous receptors and downstream pathways are known to be aberrantly activated in GC. Furthermore, several molecular alterations involving various pathways have been linked to the development and late-stage progression/metastasis of GC, and these may present novel targets for therapeutic strategies. The most important challenge for researchers in this field is to identify how to exploit the knowledge embedded in these pathways concerning the interactions among the various genes (3). Exploring pathway networks provides a suitable means to investigate how the genes interact with and regulate each other; however, the existing analytical approaches consider only the sets of genes associated with these pathways and do not take into consideration their topology, and consequently, their positions in those pathways.

At present, systems biology offers an effective approach to identify molecular mechanisms and connections between genes, and their pathways of dynamic networks (4). Systems biology approaches, which dissect the molecular mechanisms and pathways that regulate the progression of $\mathrm{GC}$, are still in their infancy. In the present study, microarray data, pathway enrichment and network topological analyses were used to identify major pathways associated with the development of GC, and to construct pathway networks and functional modules, which are based on the key pathways. The characterization of genes and pathways associated with GC may prove to be useful for identifying potential targets for the development of novel strategies for the treatment of gastric carcinoma.

\section{Materials and methods}

Microarray data. The gene expression data set, GSE29272, was downloaded from Gene Expression Omnibus (GEO; http://www.ncbi.nlm.nih.gov/geo/). For GSE29272 analysis, 
two groups of gene expression profiles, gastric cardia adenocarcinomas (GCAs; $n=62$ ) and matched normal tissues from patients, and gastric non-cardia adenocarcinomas (GNCAs; $n=72$ ) and matched normal tissues from patients, were included, based on the GPL96 Affymetrix human genome U133A array (HG-U133A; Affymetrix, Santa Clara CA, USA). The original CEL files and the platform probe annotation information file were used for the bioinformatics analysis.

Identification of differentially expressed genes (DEGs). The raw data were converted into the identifiable format using the package affy of R 2.8.2, and missing values were subsequently inserted (5). The robust MultiArray average method (6) was applied to perform background correction and data normalization, using defaulted parameters in the affy package (7). Subsequently, a differential analysis between GCA (or GNCA) and the control was performed using the limma package (8), a modified version of the standard t-test incorporating the Benjamini-Hochberg (BH) multiple hypotheses correction technique (9). DEGs were defined as the false discovery rate (FDR) $\mathrm{q}$ value $<0.05$, and adjusted $\mathrm{P}<0.01$ and fold change $\geq 2$ were set as the cut-off parameters to screen out any significant increases or decreases in gene expression levels.

Biological function annotation and enrichment analysis. In order to identify biological functions, which were disrupted in GC, a gene ontology (GO) functional enrichment analysis was performed for the DEGs using BiNGO (version 3.0.2) (10) in the open source bioinformatics software platform, Cytoscape 2.8.0 (http://www.cytoscape.org/), with a threshold of $\mathrm{P}<0.001$. BiNGO is a tool to determine which $\mathrm{GO}$ categories are statistically over-represented in a set of genes or a subgraph of a biological network.

Pathway analysis. JEPETTO plug-in (version 1.3.1) in Cytoscape (11) was used to analyze a set of DEGs associated with GC. JEPETTO offers two types of analysis: The enrichment analysis identifies pathways closely associated with a query gene set in the context of an interaction network, whereas topology analysis identifies pathways which share a similar set of topological features. EnrichNet (12) and PathExpand (13) web servers were used for the enrichment analysis. The relevant pathways from Kyoto Encyclopedia of Genes and Genomes (KEGG; http://www.genome.jp/kegg/) were identified using the XD-score method, which uses a random walk to score how close a pathway and the gene set are in the molecular interaction network.

The enrichment analysis was complemented with a network topology analysis, which was performed with TopoGSA (http://www.topogsa.net/) (14). A network analysis of biological systems revealed the connectivity and interactions of proteins associated with different cellular processes. The topology analysis identified pathways sharing a similar set of topological features. The visualization of the reconstructed pathway network was performed using the Cytoscape software.

Pathway-network construction and network modularization. A pathway clustering analysis was performed on the DEGs to
Table I. Markedly associated pathways identified in KEGG.

\begin{tabular}{lccc}
\hline Pathway/process & XD-score & q-value & $\begin{array}{c}\text { Overlap/ } \\
\text { Size }\end{array}$ \\
\hline ECM-receptor interaction & 0.810 & 0.000 & $16 / 77$ \\
Nitrogen metabolism & 0.763 & 0.081 & $3 / 14$ \\
Linoleic acid metabolism & 0.633 & 0.416 & $2 / 11$ \\
\hline
\end{tabular}

The XD-score determines the significance of the association and the q-value determines the significance of the overlap (Fisher test). The final column illustrates the number of overlapping genes compared with the size of the pathway. ECM, extracellular matrix; KEGG, Kyoto Encyclopedia of Genes and Genomes.

construct a pathway network, using the (ClueGO + CluePedia) plug-in (version 1.4) in Cytoscape $(15,16)$, according to KEGG (accessed on 14/3/2014). Using the plug-in, MCODE, (version 1.4.0; http://apps.cytoscape.org/apps/MCODE) in Cytoscape (17), the modules were identified from the network. Each module was scored using Cytoscape, according to density and size; a higher score represented a tighter module. The threshold score was set at 3.0. Within the modules, pathways were analyzed using the ClueGO plug-in (version 1.4) in Cytoscape, according to KEGG (accessed on 14/3/2014). Only pathways with the overview 'true' were considered to be positive.

\section{Results}

Identification of DEGs. The original microarray data may be systematically biased for various reasons, including the efficiency of the RNA extraction process, reverse transcription, label incorporation, exposure and spot detection (18). Therefore, data pre-processing was performed and this revealed that the fluctuations following normalization were less marked compared with those occurring prior to normalization (Fig. 1).

To obtain results with a high level of confidence, the gene expression data were analyzed by a modified standard t-test, based on the $\mathrm{BH}$ algorithm FDR method. The overlapping genes from this set were subsequently selected for further analysis. At an adjusted $\mathrm{P}=0.05,249$ genes revealed significantly different levels of expression. Specifically, 150 genes were revealed to be upregulated, whereas 99 genes were downregulated.

Pathway analysis. Pathways with the overview 'true' were considered to be positive. Ultimately, the eight most significant pathways from the groups with $\mathrm{P}<0.01$ were identified using pathway clustering analysis of the KEGG pathway datasets (data not shown). In order to further screen the most relevant pathways, an enrichment analysis was used to identify the key pathways. Three pathways or processes, extracellular matrix (ECM)-receptor interaction, nitrogen metabolism and linoleic acid metabolism, were selected, according to the XD-score significance threshold (Table I). A Fisher test q-value (P-value adjusted by false discovery rate) of 0.05 was considered as a restriction to compensate for uncertainty in the model 
Table II. Top 10 closest topological matches found in KEGG.

\begin{tabular}{lll}
\hline Pathway/process & \multicolumn{1}{c}{ Class } & Score \\
\hline Regulation of autophagy & Cellular process & 0.07 \\
Taste transduction & Cellular process & 0.07 \\
Pyrimidine metabolism & Metabolism & 0.13 \\
Calcium signaling pathway & Environmental information processing & 0.15 \\
PPAR signaling pathway & Cellular process & 0.17 \\
Hedgehog signaling pathway & Environmental information processing & 0.17 \\
Cholera infection & Human disease & 0.18 \\
Starch and sucrose metabolism & Metabolism & 0.18 \\
Purine metabolism & Metabolism & 0.19 \\
Neuroactive ligand receptor interaction & Environmental information processing & 0.22 \\
\hline
\end{tabular}

The distance score associated with each match was normalized against the sum of ranks computed from differences between the topological properties. KEGG, Kyoto Encyclopedia of Genes and Genomes; PPAR, peroxisome proliferator-activated receptor.

A

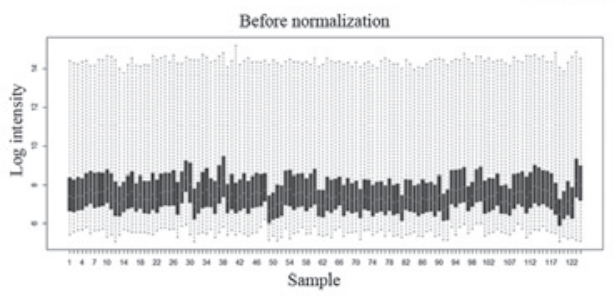

GNCA and normal



GCA and normal


B

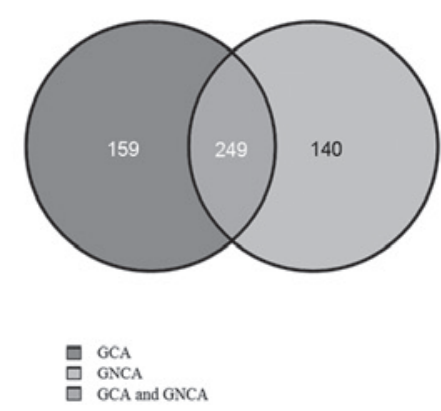

Figure 1. (A) Box plot highlighting the differences typically observed between prior to and following normalization. (B) The Venn diagram illustrates the number of differentially expressed genes identified from the two groups. GCA, gastric cardia adenocarcinoma; GNCA, gastric non-cardia adenocarcinoma.

parameters (Fig. 2A). The ECM-receptor interaction pathway appeared to be a highly enriched pathway, based on the results obtained $(X D$-score $=0.810$; q-value $=0.000)$.

In the initial step of the enrichment analysis with JEPETTO, the target gene set was mapped onto the string interaction network. Of the 249 genes identified, 164 were successfully mapped, and these were used in the further analyses.

As the input, the largest connected component (1,583 nodes) of the enriched network was analyzed. The topological properties of the network were compared with the properties of random interaction networks of an identical size. The results revealed marked differences in the topological signatures (data not shown).

The topological signature of the interactions in the enriched network was subsequently compared with those of known pathways and biological processes. The KEGG database was searched for the closest topological matches (Fig. 2B). The most similar biological mechanisms identified are listed in Table II. The closest topological matches were identified for taste transduction and the regulation of autophagy (score $=0.07)$.

Construction of the interaction pathway network and module analysis. In view of the enrichment analysis, all pathways associated with GC were used to build the network with DEGs. If a gene was identified in $\geq 2$ terms, it was assigned $\geq 2$ colors. From the network mapping, it was observed that the majority of the genes were associated with different pathways. Genes, including those encoding thrombospondin 1 (THBS1), fibronectin 1 (FN1), Myb proto-oncogene protein, and collagen type 4 variants $\alpha$ (COL14A) $1 / 2$, were involved in various processes. Furthermore, those encoding interleukin 8 , vascular cellular adhesion molecule 1, aldehyde dehydrogenase 3 family member A1, aldehyde dehydrogenase $1 \mathrm{C}$ and carbonic anhydrase 2, were involved in disparate pathways.

Using the MCODE plug-in in Cytoscape, the global network was partitioned into seven modules, which were 
Table III. Network modules and enriched signaling pathways.

\begin{tabular}{|c|c|c|c|c|}
\hline Cluster & Score $^{a}$ & Nodes & Edges & Node ID \\
\hline 1 & 8.222 & 10 & 39 & $\begin{array}{l}\text { FN1, COL4A2, THBS1, SPP1, COL4A1 } \\
\text { ECM-receptor interaction, focal adhesion, PI3K-Akt signaling pathway, } \\
\text { amoebiasis, protein digestion and absorption }\end{array}$ \\
\hline 2 & 5.250 & 9 & 21 & $\begin{array}{l}\mathrm{C} 1 \mathrm{R}, \mathrm{C} 1 \mathrm{~S}, \mathrm{C} 2, \mathrm{C} 4 \mathrm{~A}, \mathrm{C} 4 \mathrm{~B} \\
\text { Pertussis, prion diseases, complement and coagulation cascades, Staphylococcus } \\
\text { aureus infection }\end{array}$ \\
\hline 3 & 4.501 & 5 & 9 & $\begin{array}{l}\text { AKR1B10, AKR1B1 } \\
\text { Fructose and mannose metabolism, glycerolipid metabolism, pentose and } \\
\text { glucuronate interconversions }\end{array}$ \\
\hline 4 & 4.001 & 4 & 6 & $\begin{array}{l}\text { Metabolism of xenobiotics by cytochrome P450, retinol metabolism, fatty } \\
\text { acid degradation, chemical carcinogenesis }\end{array}$ \\
\hline 5 & 3.600 & 6 & 9 & $\begin{array}{l}\text { ALDH3A1, ALDH3B1, ALDH1A3, ALDH3B2 } \\
\text { drug metabolism, glycolysis/gluconeogenesis }\end{array}$ \\
\hline 6 & 3.500 & 5 & 7 & $\begin{array}{l}\text { MAOB, MAOA } \\
\text { Histidine metabolism, } \beta \text {-alanine metabolism, phenylalanine metabolism }\end{array}$ \\
\hline 7 & 3.000 & 3 & 3 & HBB, African trypanosomiasis, malaria \\
\hline
\end{tabular}

${ }^{a}$ Calculated by density x nodes. ID, identifier; FN1, fibronectin 1; THBS1, thrombospondin 1; SPP1, secreted phosphoprotein 1; COL4A, collagen type 4 A; ECM, extracellular matrix; PI3K, phosphoinositide 3-kinase; C1R, complement R subcomponent; C1S, complement S subcomponent; $\mathrm{C} 2$, complement component 2; C4A/B, complement component 4A/B; AKR1B, aldo-keto reductase 1B family member; ALDH, aldehyde dehydrogenase; MAOB/A, monoamine oxidase A/B; HBB, hemoglobin $\beta$.

A

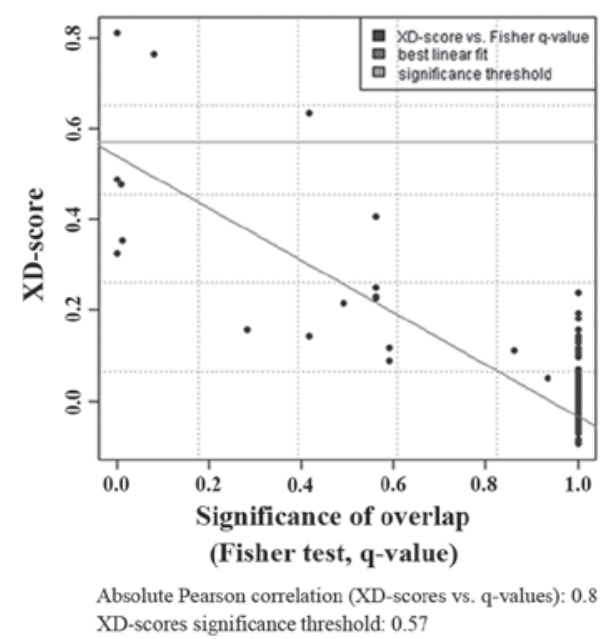

B

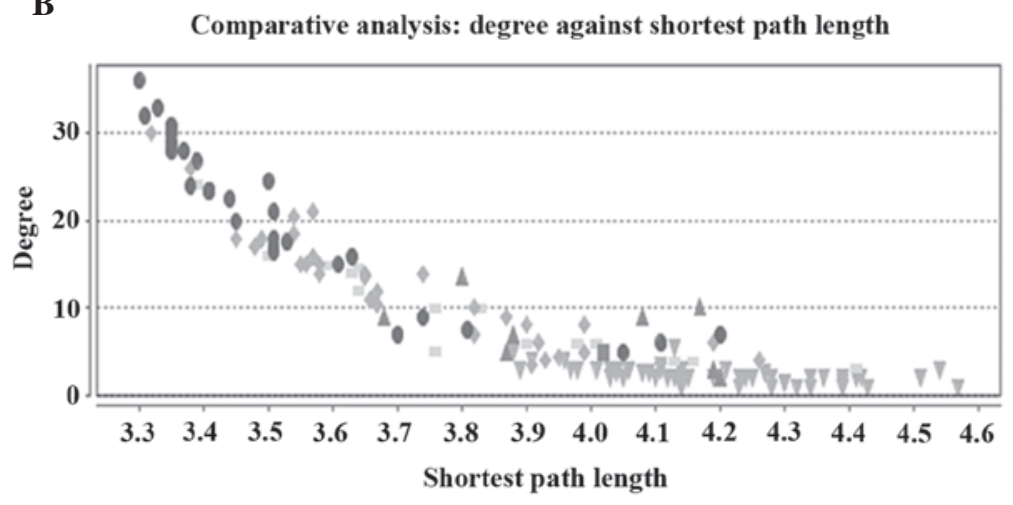

II Uploaded gene set (median) Human diseases $\Delta$ Genetic information processing

$\downarrow$ Cellular processes $=$ Environmental information processing $\nabla$ Metabolism

Figure 2. (A) A JEPETTO screen-shot of the regression plot between the XD-scores and the overlap significance (q-value). Each dot in the regression plot represented a pathway or process predicted in the enrichment analysis, in terms of the degree of association with differentially expressed genes. The best linear fit was revealed by the line intersecting the dots. The XD-score significance threshold was demonstrated by the grey horizontal line. (B) A JEPETTO results panel is shown, which illustrates a comparative analysis of the topological properties. The target network is represented with a square.

regarded as the network core in terms of functionality (Table III). To determine the biological function of each module, pathway enrichment from the seven modules was processed using the ClueGO plug-in and the KEGG database. Although numerous pathways in the KEGG database were detected, only those with the overview 'true' (positive) were selected. A total of 22 pathways distributed in seven modules were classified as being enriched. Since the pathway within a module represents only a part of the signal cascade network, the module including the ECM-receptor interaction pathway achieved the highest score (8.222). The scores represent the module density, and the denser modules are ranked more highly.

Cluster 1 from seven modules was demonstrated to be highly enriched. The ECM-receptor interaction pathway had a significant level of overlap with other pathways, focal 




Figure 3. (A) A functionally grouped network with pathways and differentially expressed genes is schematically shown (genes are represented by their approved HUGO Gene Nomenclature Committee symbols). (B) A network centred on the ECM-receptor interaction pathway (cluster 1) is illustrated, together with the gene inter-associations. The terms are linked based on the $\kappa$ score $(\geq 0.3)$. The edge thickness is scaled between the minimum and maximum scores demonstrated. The genes, which are upregulated or downregulated are identified by squares. Arrows represent the activation and the inhibition symbol represents inhibition. Emboldened lines indicate a binding interaction. COL1/2/3/4/5Ax, collagen type 1 (or type 2, 3, 4 or 5) family A member x; PI3K, phosphoinositide 3-kinase; THBS, thrombospondin; ECM, extracellular matrix; FN, fibronectin; SPP, secreted phosphoprotein; MYB, Myb proto-oncogene protein; LAMB, laminin $\beta$; GSTA, glutathione S-transferase $\alpha$; VCAN, versican.

adhesion, phosphoinositide 3-kinase (PI3K)-Akt signaling pathway, amoebiasis, protein digestion and absorption. Considerable crosstalk existed among them and certain DEGs, FN1, secreted phosphoprotein 1, COL4A1, COL4A2 and THBS1 (Fig. 3B). Numerous, close interactions were identified between pathways and relevant genes in this module.

\section{Discussion}

Systems biology, which is defined as the systematic study of complex regulation and interaction in biological systems, assesses cellular processes from a systematic, rather than a reductionistic perspective. (19). In the present study, a systems biology approach was performed to identify any DEGs associated with GC, and to predict the underlying molecular mechanisms.

In the initial stage of the enrichment analysis with JEPETTO, ECM-receptor interaction pathways appeared at the top of the pathways ranking. The high XD-score of 0.810 was $>1.5$ times higher compared with the significance threshold of 0.57 identified by the regression fit. Among the other top-ranked pathways and processes were nitrogen metabolism and linoleic acid metabolism. These two pathways had a Fisher test q-value $>0.05$ and a small overlap size. The average 
shortest path length was identical with the size in random networks, and the node degree was slightly higher compared with the average node degree, which further revealed certain dense interactions between the DEGs. However, the results may also support the hypothesis that the target network is specific, and that interactions between DEGs are unlike those typically observed in the background or random network as a whole. The closest topological match was identified for regulation of autophagy. Autophagy is dysregulated in a wide spectrum of human cancer types (20). There is mounting evidence to suggest that alterations in autophagy-associated genes are associated with the pathogenesis of gastrointestinal cancer. Frameshift mutations in the autophagy-related protein (ATG)2B, ATG5, ATG9B and ATG12 genes with mononucleotide repeats are common, indicating that the genetic disruption of autophagy may contribute to gastrointestinal tumorigenesis (21). The decrease in autophagic capacity may be associated with tumorigenesis and the development of GC.

In the constructed pathway map, several genes were involved in tumorigenesis. To assess the decomposition in the clusters of a network, modularization methods were used to further dissemble the networks. From cluster 1, certain pathways associated with GC, including the PI3K-Akt signaling pathway, were also enriched. The ECM-receptor interaction pathway, in association with the PI3K-Akt signaling, focal adhesion, amoebiasis, and protein digestion and absorption pathways (Fig. 3), were considered as the key pathways mediating the actions of GC. In addition, associated DEGs were derived from an identical module. The genetic interactions between the DEGs and these pathways were also displayed to confirm possible connections.

ECM is a macromolecular network, comprising collagens, non-collagenous glycoprotein, glycosaminoglycan, proteoglycans, elastin and other components. The ECM was revealed to influence cell survival, death, proliferation and differentiation, as well as cancer metastasis (22). Collagen is the major constituent of the tumor ECM, and several types of collagens have been implicated in the focal adhesion and ECM-receptor interaction pathways in gastric carcinoma (23).

Focal adhesions not only provide structural links between the ECM and the actin cytoskeleton, they also comprise important sites of signal transduction pathways leading to various physiological and pathological processes, including cancer (24). At the molecular level, focal adhesions are predominantly mediated by integrins, which have been identified as exerting a critical role in the invasion and metastasis of cancer (25). Kindlin-2 is a member of the focal adhesion protein family recruited to integrin-containing adhesion sites. Kindlin-2 occupied an important role in the progression of GC and was an independent risk factor of progression-free survival (26). Few reports have focused on the connections between the protein digestion and absorption pathway, and GC. Although acidic and non-acidic gastric exocrine secretion are required for micronutrient absorption and protein digestion, ingested proteins undergo a complex series of degradative processes following the action of gastric acid (27). Disorders of the protein digestion and absorption pathway lead to a decrease in gastric exocrine secretion with a possible progression to mucosal atrophy, which may ultimately lead to cancer.
The majority of the unregulated genes in GC, including COL1A1, COL2A1, COL4A1 and COL4A2, are associated with cell adhesion or migration and the ECM-receptor interaction pathways (28). Previous studies have demonstrated that the overexpression of COL1A1 and COL1A2 is associated with tumor invasion, metastasis and stage grouping, indicating that these genes may be novel genetic markers for high-grade malignancy $(29,30)$. In addition, THBS1 is a multifunctional protein implicated in cancer cell adhesion, migration, invasion, inhibition of angiogenesis and activation of latent transforming growth factor $\beta$. Furthermore, THBS1 is an inhibitor of angiogenesis with tumor suppressor properties $(31,32)$. A polymorphism in the 5'-untranslated region of THBS1 (rs1478604 A>G) is reported to be associated with lymph node metastasis of GC (33). FN1 is an ECM protein, which mediates the activation of focal adhesion kinase, which promotes cell motility through the extracellular signal-regulated kinase or PI3K/Akt signaling pathways to upregulate matrix metalloproteinase 9 (MMP 9)/calpain-2 or MMP 9/RhoA activity (34). Notably, several downregulated genes are associated with GC, including COL3A1 and COL5A1. The incidence of GC is likely to be associated with acute changes in the expression of these genes.

In conclusion, the present study demonstrated that changes in the primary pathways (i.e. the focal adhesion, PI3K-Akt signaling, amoebiasis, protein digestion and absorption, and ECM-receptor interaction pathways) may be associated with GC. Extensive connections between these pathways and their candidate genes, which may be associated with GC, were also identified by modularity analysis. These findings shed new light on the biology of GC, and provided novel insights into an improved understanding of the molecular basis for identifying the associations between candidate genes and key pathways.

\section{Acknowledgements}

This study was supported by the General Program of the National Natural Science Foundation of China (no. 81373097). The authors would like to thank the focus group participants and volunteers from community organization partners for their time and efforts.

\section{References}

1. Catalano V, Labianca R, Beretta GD, Gatta G, de Braud F and Van Cutsem E: GC. Crit Rev Oncol Hematol 71: 127-164, 2009.

2. Crew KD and Neugut AI: Epidemiology of gastric cancer. World J Gastroenterol 12: 354-362, 2006.

3. Draghici S, Khatri P, Tarca AL, Amin K, Done A, Voichita C, Georgescu C and Romero R: A systems biology approach for pathway level analysis. Genome Res 17: 1537-1545, 2007.

4. Loscalzo J and Barabasi AL: Systems biology and the future of medicine. Wiley Interdiscip Rev Syst Biol Med 3: 619-627, 2011.

5. Troyanskaya O, Cantor M, Sherlock G, Brown P, Hastie T, Tibshirani R, Botstein D and Altman RB: Missing value estimation methods for DNA microarrays. Bioinformatics 17: 520-525, 2001

6. D'Souza M, Zhu X and Frisina RD: Novel approach to select genes from RMA normalized microarray data using functional hearing tests in aging mice. J Neurosci Methods 171: 279-287, 2008.

7. Davis JW: Bioinformatics and computational biology solutions using R and bioconductor. J Am Statistical Association 102: 388-389, 2007. 
8. Diboun I, Wernisch L, Orengo CA and Koltzenburg M: Microarray analysis after RNA amplification can detect pronounced differences in gene expression using limma. BMC Genomics 7: 252, 2006

9. Benjamini Y, Drai D, Elmer G, Kafkafi N and Golani I: Controlling the false discovery rate in behavior genetics research. Behav Brain Res 125: 279-284, 2001.

10. Maere S, Heymans K and Kuiper M: BiNGO: A cytoscape plugin to assess overrepresentation of gene ontology categories in biological networks. Bioinformatics 21: 3448-3449, 2005.

11. Winterhalter C, Widera P and Krasnogor N: JEPETTO: A Cytoscape plugin for gene set enrichment and topological analysis based on interaction networks. Bioinformatics 30 1029-1030, 2014

12. Glaab E, Baudot A, Krasnogor N, Schneider R and Valencia A: EnrichNet: Network-based gene set enrichment analysis. Bioinformatics 28: i451-i457, 2012.

13. Glaab E, Baudot A, Krasnogor N and Valencia A: Extending pathways and processes using molecular interaction networks to analyse cancer genome data. BMC Bioinformatics 11: 597, 2010

14. Glaab E, Baudot A, Krasnogor N and Valencia A: TopoGSA Network topological gene set analysis. Bioinformatics 26: 1271-1272, 2010.

15. Bindea G, Galon J and Mlecnik B: CluePedia cytoscape plugin: Pathway insights using integrated experimental and in silico data. Bioinformatics 29: 661-663, 2013.

16. Bindea G, Mlecnik B, Hackl H, Charoentong P, Tosolini M Kirilovsky A, Fridman WH, Pagès F, Trajanoski Z and Galon J: ClueGO: A cytoscape plug-in to decipher functionally grouped gene ontology and pathway annotation networks. Bioinformatics 25: 1091-1093, 2009.

17. Yu X and Sun S: Comparing a few SNP calling algorithms using low-coverage sequencing data. BMC Bioinformatics 14: 274, 2013

18. Alakwaa FM, Solouma NH and Kadah YM: Construction of gene regulatory networks using biclustering and Bayesian networks. Theor Biol Med Model 8: 39, 2011.

19. Mitra S, Das S and Chakrabarti J: Systems biology of cancer biomarker detection. Cancer Biomark 13: 201-213, 2013.

20. Zhang L, Sung JJ, Yu J, Ng SC, Wong SH, Cho CH, Ng SS, Chan FK and Wu WK: Xenophagy in Helicobacter pylori- and Epstein-Barr virus-induced gastric cancer. J Pathol 233: 103-112, 2014.

21. Kang MR, Kim MS, Oh JE, Kim YR, Song SY, Kim SS, Ahn CH Yoo NJ and Lee SH: Frameshift mutations of autophagy-related genes ATG2B, ATG5, ATG9B and ATG12 in gastric and colorectal cancers with microsatellite instability. J Pathol 217: 702-706, 2009.

22. Bijian K, Takano T, Papillon J, Khadir A and Cybulsky AV: Extracellular matrix regulates glomerular epithelial cell survival and proliferation. Am J Physiol Renal Physiol 286: F255-F266, 2004.
23. Yin Y, Zhao Y, Li AQ and Si LM: Collagen: A possible prediction mark for gastric cancer. Medical Hypotheses 72: $163-165,2009$

24. Yam JW, Tse EY and Ng IO: Role and significance of focal adhesion proteins in hepatocellular carcinoma. J Gastroenterol Hepatol 24: 520-530, 2009.

25. Ramos DM, But M, Regezi J, Schmidt BL, Atakilit A, Dang D, Ellis D, Jordan R and Li X: Expression of integrin beta 6 enhances invasive behavior in oral squamous cell carcinoma. Matrix Biol 21: 297-307, 2002.

26. Shen Z, Ye Y, Dong L, Vainionpää S, Mustonen $H$, Puolakkainen P and Wang S: Kindlin-2: A novel adhesion protein related to tumor invasion, lymph node metastasis and patient outcome in gastric cancer. Am J Surg 203: 222-229, 2012.

27. Colacci E, Pasquali A and Severi C: Exocrine gastric secretion and gastritis: Pathophysiological and clinical relationships. Clin Ter 162: e19-25, 2011.

28. Jinawath N, Furukawa Y, Hasegawa S, Li M, Tsunoda T, Satoh S, Yamaguchi T, Imamura H, Inoue M, Shiozaki H and Nakamura Y: Comparison of gene-expression profiles between diffuse- and intestinal-type gastric cancers using a genome-wide cDNA microarray. Oncogene 23: 6830-6844, 2004.

29. Ossandon FJ, Villarroel C, Aguayo F, Santibanez E, Oue N, Yasui $\mathrm{W}$ and Corvalan AH: In silico analysis of gastric carcinoma serial analysis of gene expression libraries reveals different profiles associated with ethnicity. Mol Cancer 7: 22, 2008.

30. Ramaswamy S, Ross KN, Lander ES and Golub TR: A molecular signature of metastasis in primary solid tumors. Nat Genet 33: 49-54, 2003.

31. Wiedemann S, Wessela T, Schwarz K, Joachim D, Jercke M, Strasser RH, Ebner B and Simonis G: Inhibition of anti-apoptotic signals by Wortmannin induces apoptosis in the remote myocardium after LAD ligation: Evidence for a protein kinase C-delta-dependent pathway. Mol Cell Biochem 372: 275-283, 2013.

32. Adams JC: Functions of the conserved thrombospondin carboxy-terminal cassette in cell-extracellular matrix interactions and signaling. Int J Biochem Cell Biol 36: 1102-1114, 2004.

33. Lin XD, Chen SQ, Qi YL, Zhu JW, Tang Y and Lin JY: Polymorphism of THBS1 rs1478604 A>G in 5-untranslated region is associated with lymph node metastasis of gastric cancer in a Southeast Chinese population. DNA Cell Biol 31: 511-519, 2012.

34. Rosman DS, Phukan S, Huang CC and Pasche B: TGFBR1*6A enhances the migration and invasion of MCF-7 breast cancer cells through RhoA activation. Cancer Res 68: 1319-1328, 2008 . 